\title{
Optimization of Modified Sliding Mode Controller for an Electro-hydraulic Actuator System with Mismatched Disturbance
}

\author{
Siti Marhainis Othman ${ }^{1}$, M. F. Rahmat ${ }^{2}$, S. M. Rozali ${ }^{3}$, Zulfatman Has ${ }^{4}$, A. F. Z. Abidin ${ }^{5}$ \\ ${ }^{1,2}$ Department of Control and Mechatronics Engineering, Faculty of Electrical Engineering, \\ Universiti Teknologi Malaysia, Malaysia \\ ${ }^{1}$ Mechatronic Engineering Programme, School of Mechatronic Engineering, Universiti Malaysia Perlis \\ ${ }^{3,5}$ Department of Electrical Engineering Technology, Faculty of Engineering Technology, \\ Universiti Teknikal Malaysia Melaka, Malaysia \\ ${ }^{4}$ Dept. of Electrical Engineering, University of Muhammadiyah Malang, Kota Malang, Indonesia
}

\section{Article Info \\ Article history: \\ Received Aug 28, 2017 \\ Revised Feb 14, 2018 \\ Accepted Apr 4, 2018 \\ Keyword: \\ Electro-hydraulic actuator Gravitational particle swarm Gravitational search algorithm Modified sliding mode control Particle swarm optimization Sum square error}

\begin{abstract}
This paper presents the design of the modified sliding mode controller (MSMC) for the purpose of tracking the nonlinear system with mismatched disturbance. Provided that the performance of the designed controller depends on the value of control parameters, gravitational search algorithm (GSA), and particle swarm optimization (PSO) techniques are used to optimize these parameters in order to achieve a predefined system's performance. In respect of system's performance, it is evaluated based on the tracking error present between reference inputs transferred to the system and the system output. This is followed by verification of the efficiency of the designed controller in simulation environment under various values, with and without the inclusion of external disturbance. It can be seen from the simulation results that the MSMC with PSO exhibits a better performance in comparison to the performance of the similar controller with GSA in terms of output response and tracking error.
\end{abstract}

Copyright () 2018 Institute of Advanced Engineering and Science. All rights reserved.

\section{Corresponding Author:}

M. F. Rahmat,

Department of Control and Mechatronics Engineering,

Faculty of Electrical Engineering,

Universiti Teknologi Malaysia, Malaysia.

Email: fuaad@fke.utm.my

\section{INTRODUCTION}

Electro-Hydraulic Actuator (EHA) system is one of the important drive systems in industrial sectors and most engineering practices. However, the system is highly nonlinear due to many factors, such as leakage, friction, and especially the expression of fluid flow through the servo valve [12]. With these attributes being present, the system's performance will be reduced significantly. In order to overcome the issue previously highlighted, the controller utilized for the system should be robust enough to overcome the entire operating range. Therefore, a suitable controller needs to be designed for a positive performance to be shown by the electro-hydraulic actuator.

The raised numbers of works conducted with electro-hydraulic actuator system have been proposed over the past decades ranged from linear control, nonlinear control to intelligent control strategies such as [3]-[6] have been proposed over the past decades. Based on the literature study in [7]-[10], sliding mode control (SMC) is found to be efficient and widely applied in nonlinear systems [11]. However, it has been noticed that most of the existing results regarding the sliding surface design are more emphasized on the matched uncertainties and disturbances attenuation, provided the insensitivity of the sliding motion of 
traditional SMC to matched uncertainties and disturbance [12]. This indicates the presence of uncertainties and disturbances in the same channel as the channel of the control input. Despite this indication, the presence of these uncertainties in various practical systems may not be able to fulfil the well-known matching condition. In this study, the dynamic model and design requirement of electro-hydraulic actuator were obtained from National Institute for Aerospace Research, Romania [13]. As for the dynamic model used in this study, the track input disturbance took place on a different channel from the channel of the control input. It can be gathered that with mismatched system, not only the sliding motion of traditional SMC is severely affected by the mismatched disturbance, SMC is no longer capable of coping, in spite of its well-known robustness Given the significance of attenuating mismatched uncertainties and disturbances in practical applications, many authors have devoted themselves to designing the sliding surface for uncertain systems with mismatched disturbance. Additionally, a few number of literatures have used conventional SMC with some modification in its sliding surface[14]-[16]. These modifications are done to enhance the ability of the modified SMC (MSMC).

It is also important to emphasise that estimation is seen as one way to improve the accuracy of MSMC. Therefore, the results which display the influence which will be posed by the parameters of the designed controller on the tracking performance will be shown in one of the sections in this paper. According to reviews, particle swarm optimization (PSO) is the most-applied technique for combination with sliding mode control in order to adjust its control parameters. Furthermore, gravitational search algorithm (GSA) is a relatively new nature-based optimization algorithm, which is in accordance to the law of gravity [17], [18]. However, both algorithm have not been specifically applied in the estimation of MSMC controller's parameter for mismatched disturbance system, such as an electro-hydraulic actuator which has been used in this study. In fact, there has not been any researcher who has devoted themselves to conducting parameters estimation for MSMC for the improvement of its accuracy and performance.

This paper presents an investigation into performance comparison between MSMC, which has been optimized using PSO and GSA. Sum Square Error (SSE) has been used as the objective function for obtaining the optimum value of the controller parameters. Essentially, a good tracking response will provide a small SSE value. Furthermore, comparative assessment of both optimization method for the system's performance has been presented and discussed. Moreover, the main contents of this paper are summarized as follows: Section 2 illustrates the mathematical modelling of the developed system. Following that, the MSMC algorithm derivation is demonstrated in Section 3. Then, the discussion on optimization algorithms used in this study is presented in section 4. Observation results are discussed, compared, and presented in Section 5. Last but not least, summary and conclusions are presented in Section 7.

\section{RESEARCH METHOD}

\subsection{EHA system modelling}

The actuator dynamic equation of electro-hydraulic actuator servo system is expressed as[13]. As a result, the differential equations which govern the dynamics of electro-hydraulic actuator servo system with external disturbance injected to its actuator is constructed as

$$
\begin{aligned}
& \dot{x}_{1}=x_{2} \\
& \dot{x}_{2}=-\frac{k}{m} x_{1}-\frac{f}{m} x_{2}+\frac{s}{m} x_{3}-\frac{F_{L}}{m} \\
& \dot{x}_{3}=-\frac{s}{k} x_{2}-\frac{k_{l}}{k_{c}} x_{3}+\frac{c}{k_{c}} \sqrt{\frac{P_{a}-x_{3}}{2}} k_{v}
\end{aligned}
$$

The system equations can be expressed as a product of matrix such as:

$$
\left[\begin{array}{c}
\dot{x_{1}} \\
\dot{x_{2}} \\
\dot{x_{3}}
\end{array}\right]=\left[\begin{array}{ccc}
0 & 1 & 0 \\
-\frac{k}{m} & -\frac{f}{m} & \frac{s}{m} \\
0 & -\frac{s}{k_{c}} & -\frac{k_{l}}{k_{c}}
\end{array}\right]\left[\begin{array}{l}
x_{1} \\
x_{2} \\
x_{3}
\end{array}\right]+\left[\begin{array}{c}
0 \\
0 \\
\frac{c}{k_{c}} \sqrt{\frac{P_{a}-x_{3}}{2}}
\end{array}\right] u+\left[\begin{array}{c}
0 \\
-\frac{F_{L}}{m} \\
0
\end{array}\right]
$$

where $x_{1}$ represents the displacement of the load while $x_{2}$ indicates load velocity and $x_{3}$ represents the differential pressure $\rho_{1}-\rho_{2}$ between the cylinder chambers caused by the load. Meanwhile, $F_{L}$ represents the external disturbance inflicted on the system and it is possible for it to be a constant or a time varying signal. This system is a mismatched system due to the disturbance it inflicts on different channels from the control 
input $u$.Table 1 display the parameters of electro hydraulic actuator servo system, which are represented by (1), (2) and (3).

Table 1. Parameter of EHA Servo System

\begin{tabular}{lcc}
\hline \multicolumn{1}{c}{ Parameters } & Value & Unit \\
\hline Load at the EHA rod $(m)$ & 0.33 & $\mathrm{Ns} / \mathrm{cm}^{2}$ \\
Piston Area $(S)$ & 10 & $\mathrm{~cm}^{2}$ \\
Coefficient of viscous friction $(f)$ & 27.5 & $\mathrm{Ns} / \mathrm{cm}$ \\
Coefficient of aerodynamic elastic force $(k)$ & 1000 & $\mathrm{~N} / \mathrm{cm}$ \\
Valve port width $(w)$ & 0.05 & $\mathrm{~cm}$ \\
Supply pressure $\left(P_{a}\right)$ & 2100 & $\mathrm{~N} / \mathrm{cm}^{2}$ \\
Coefficient of volumetric flow of the valve port $\left(c_{d}\right)$ & 0.63 & - \\
Coefficient of internal leakage $\left(k_{l}\right)$ & $2.38 \times 10^{-3}$ & $\mathrm{~cm}^{5} / \mathrm{Ns}$ \\
Coefficient of servo valve $\left(k_{v}\right)$ & 0.017 & $\mathrm{~cm}^{5} /$ \\
Coefficient involving bulk modulus and EHA volume $\left(k_{c}\right)$ & $2.5 \times 10^{-4}$ & $\mathrm{~cm}^{5} / \mathrm{N}$ \\
Oil density $(\rho)$ & $8.87 \times 10^{-7}$ & $\mathrm{Ns} / \mathrm{cm}^{4}$ \\
\hline
\end{tabular}

\subsection{Controller design}

The study of sliding mode control has gained popularity in recent years as a methodology for controlling nonlinear systems with modelling uncertainties and external disturbances. It is known that the crucial and the most important step of SMC design is the construction of sliding surface $S(t)$ which is expected to response desired control specifications and performance. The trajectories are enforced to lie on the sliding surface. Given the desired position trajectory as $x_{d}=\left[x_{1 d}, x_{2 d}, x_{3 d}\right]^{T} \in R^{n}$, and defined $\dot{x}_{1 d}=x_{2 d}, \quad \dot{x}_{2 d}=x_{3 d}$. Vector of the system states are assumed measurable and defined as $x=\left[x_{1}, x_{2}, x_{3}\right]^{T}=\left[x_{p}, v_{p}, P_{L}\right] \in R^{n}$. The state error of the system is defined as

$$
e_{i}=x_{i}-x_{i d}
$$

where $i=1$ to 3 and $e \in R^{n}$.

The control objective of this design a bounded control input $u$. Hence, the output position $x_{p}$ tracks as closely as possible the desired position trajectory $x_{d}$ in spite of various model uncertainties. In order to achieve the states of the system to track the desired trajectories at the same time, the function of a new sliding surface is proposed is similar as in [14].

$$
S(t)=x_{2}+x_{3}+c_{1} e_{1}+c_{2} \int e_{1}
$$

where $c_{1}$ and $c_{2}$ are a strictly positive constant to be specified according to the desired dynamics of the closed-loop system, while $e_{1}$ is dependent to $c_{1}$ and $c_{2}$. The desired dynamic response for the system is given as $S=\dot{S}=0$ when the sliding surface is moving. If $S$ is forced to be zero, the desired dynamics is attained and the tracking error will converge to zero. Then, it can be obtained.

$$
\dot{e_{1}}=-\dot{x}_{2}-\dot{x}_{3}-c_{2} e_{1}
$$

To obtain the control law, the constant plus proportional reaching law method was applied as in [19] and [20]. The dynamics of the switching function are directly specified by this approach which is described by the reaching function of the form

$$
\dot{S}=-Q \operatorname{sign}(S)-K S
$$

where $Q$ and $K$ are a constant with positive value and $\operatorname{sign}(S)$ represents the signum function which have a piecewise function as below:

$$
\operatorname{sign}(S)=\left\{\begin{array}{cc}
1 & ; S>0 \\
0 & ; S=0 \\
-1 & ; S<0
\end{array}\right.
$$


Such that the derivative of (6) gives:

$$
\dot{S}=\dot{x_{2}}+\dot{x_{3}}+c_{1} \dot{e_{1}}+c_{2} e_{1}
$$

Substitute (5) in (10):

$$
\dot{S}=\dot{x_{2}}+\dot{x_{3}}+c_{1}\left[\dot{x_{1}}-\dot{x_{1 d}}\right]+c_{2}\left[x_{1}-x_{1 d}\right]
$$

Substitute (1), (2) and (3) in (11):

$$
\begin{aligned}
& \dot{S}=\left[-\frac{k}{m} x_{1}-\frac{f}{m} x_{2}+\frac{s}{m} x_{3}-\frac{F_{L}}{m}\right]+\left[-\frac{s}{k_{c}} x_{2}-\frac{k_{l}}{k_{c}} x_{3}+\frac{c}{k_{c}} \sqrt{\frac{P_{a}-x_{3}}{2}} k_{v} u\right]+c_{1}\left[\dot{x}_{1}-\dot{x}_{1 d}\right]+ \\
& c_{2}\left[x_{1}-x_{1 d}\right]
\end{aligned}
$$

The control law is obtained by substituting (8) in (12):

$$
\begin{aligned}
& -Q \operatorname{sgn}(S)=\left[-\frac{k}{m} x_{1}-\frac{f}{m} x_{2}+\frac{s}{m} x_{3}-\frac{F_{L}}{m}\right]+\left[-\frac{s}{k_{c}} x_{2}-\frac{k_{l}}{k_{c}} x_{3}+\frac{c}{k_{c}} \sqrt{\frac{P_{a}-x_{3}}{2}} k_{v} u\right]+c_{1}\left[\dot{x}_{1}-\dot{x}_{1 d}\right]+ \\
& c_{2}\left[x_{1}-x_{1 d}\right]
\end{aligned}
$$

Therefore,

$$
\begin{aligned}
& u=\frac{k_{c}}{c k_{v}} \sqrt{\frac{2}{P_{a}-x_{3}}}\left[-Q \operatorname{sgn}(S)-K S+\frac{k}{m} x_{1}+\frac{f}{m} x_{2}-\frac{s}{m} x_{3}+\frac{F_{L}}{m}+\frac{s}{k_{c}} x_{2}+\frac{k_{l}}{k_{c}} x_{3}-c_{1} \dot{x_{1}}+c_{1} x_{1 d}-\right. \\
& \left.c_{2} x_{1}+c_{2} x_{1 d}\right]
\end{aligned}
$$

Then, the control law in (13) can be expressed as:

$$
u=u_{n}+u_{e q}
$$

where

$$
u_{n}=\frac{k_{c}}{c k_{v}} \sqrt{\frac{2}{P_{a}-x_{3}}}\left[\begin{array}{c}
\frac{k}{m} x_{1}+\frac{f}{m} x_{2}-\frac{s}{m} x_{3}+\frac{F_{L}}{m}+\frac{s}{k_{c}} x_{2}+\frac{k_{l}}{k_{c}} x_{3}-c_{1} \dot{x_{1}}+c_{1} x_{1 d}- \\
c_{2} x_{1}+c_{2} x_{1 d}
\end{array}\right]
$$

and

$$
u_{e q}=\frac{k_{c}}{c k_{v}} \sqrt{\frac{2}{P_{a}-x_{3}}}[-Q \operatorname{sgn}(S)-K S]
$$

where $u_{n}$ is nominal control for the nominal part of the system and $u_{e q}$ is the equivalent control with switching function to ensure the robustness.

\subsection{Optimization algorithm}

In order to obtain a positive tracking performance, choice of control parameters for the designed controller is important. Therefore, the designed MSMC is integrated with PSO and GSA so that the optimum value of the control parameters can be attained. This is essential for any changes to be made on different values of external disturbance signal injected into the system. Figure 1 illustrates the block diagram of the proposed MSMC with PSO and GSA algorithm.

MSMC, which is designed for electro-hydraulic actuator servo system, consists of four control parameters namely $c_{1}, c_{2}, Q$, and $K$. The value of these parameters should be chosen in order to reduce the amount of tracking errors. With this reason, for the improvement of the adaptation characteristic of the system, PSO and GSA are used to determine the optimum value of these parameters, specifically through the assimilation of modified sliding mode controller with these three algorithms. Besides, the way this task is performed by PSO and GSA is in accordance to SSE as an objective function. Moreover, a good tracking 
response will result in a small SSE value. The formula of SSE is provided by:

$$
S S E=\sum_{k=1}^{n}\left(x_{k}-y_{r e f}\right)^{2}
$$

where $k$ represents the number of iteration, while $x_{k}$ indicates the system output at $k$ iteration and $y_{\text {ref }}$ is the reference input given to the system. Essentially, optimization algorithm is utilized with the purpose to minimize SSE value.

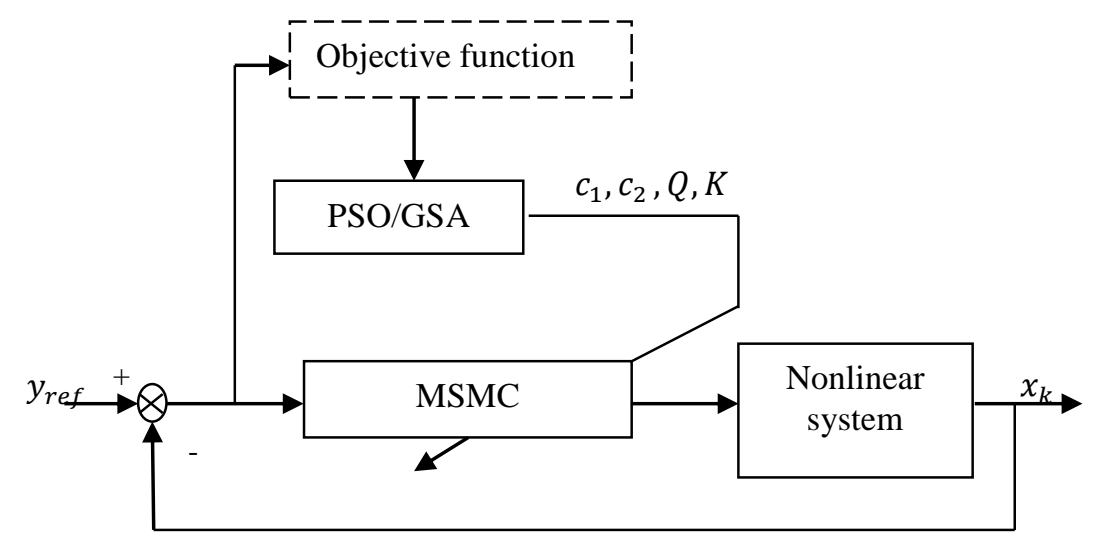

Figure 1. Block diagram of the MSMC with PSO, GSA and GPS algorithm

\subsubsection{Particle swarm optimization}

The particle swarm optimization (PSO) algorithm is a population-based search algorithm, which is in accordance to the simulation of the social behaviour of birds within a flock. It is a computational method which optimizes a problem through the effort of iteratively improving a candidate solution with regard to the given quality measure [21]. Individuals in a particle swarm emulate the success of neighbouring individuals and their own successes. Furthermore, this algorithm maintains a swarm of particles through the representation of potential solution done by each of the particles. The important parameters of PSO for this application are number of particle $(i)$, number of dimensions which represent number of control variables $(j)$, fitness, $f i t$ of global best position, $(\widehat{\boldsymbol{y}}(t))$, fitness, the $f i t$ of personal best position $\left(\boldsymbol{y}_{i}\right)$, fitness for each particle, $\left(f i t_{i}(t)\right)$, particle's position $\left(\boldsymbol{x}_{\boldsymbol{i}}\right)$, social coefficient $\left(c_{2}\right)$, cognitive coefficient $\left(c_{1}\right)$, inertia weight $(w)$, lower bound value $\left(\boldsymbol{x}_{\min }\right)$, upper bound value $\left(\boldsymbol{x}_{\max }\right)$ and number of iteration $(t)$.

\subsubsection{Gravitational search algorithm}

Gravitational Search Algorithm (GSA) is an optimization technique where its development is based on the concept of Newton theory and metaphor of gravitational kinematics. There are four specifications for each agent in this algorithm: position, inertial mass, and active and passive gravitational mass. The position of the mass corresponds to a solution of the problem. Its gravitational and inertial masses are determined by a fitness function. A particular solution is represented by each mass, and the algorithm is navigated through proper adjustment of the gravitational and inertial masses [17]. The position of the agent corresponds to a solution of the problem, and its mass is determined by using a fitness function Apart from that, the other main parameters for GSA are number of iteration $(t)$, number of dimension which represents number of control parameters $(d)$, and the best and worst fitness in population which is dependent on GSA's objective function (represented by Best and Worst respectively) On the other hand, force $\left(F_{i}^{d}(t)\right.$ ), acceleration $\left(a_{i}^{d}(t)\right)$, velocity $\left(v_{i}^{d}(t)\right)$, position $\left(x_{i}^{d}(t)\right)$, mass $\left(M_{i}(t)\right)$, gravitational constant $(G(t))$, and Euclidean distance between two masses $i$ and $j$, along $\left(R_{i j}(t)\right)$.

\section{RESULTS AND ANALYSIS}

Simulation experiment has been done using MATLAB/Simulink 2015 software. For the assessment of the controller's response, a square-shaped wave signal is chosen as a reference trajectory for electrohydraulic actuator system, as illustrated in [22]. The designed controller assists the system in tracking this signal so that smaller tracking error can be obtained. The external force, with a constant value of 
$F_{L}=10500 \mathrm{~N}$, is added as a perturbation to the system actuator. The implementation of optimization algorithms is conducted using parameters, number of particles, and the use of $i$, which represents the particles at $5,10,15,20$, and 25 . The initial value of the number of iteration $(t)$ is set at 10 , followed by its increase to $20,30,40$, and 50 iterations. In case of the presentation of results in this section, the output plot is yielded by 5 particles within 10 iterations, 15 particles within 30 iterations, and 25 particles within 50 iterations. These particles are chosen for PSO and GSA in order to observe the performance of the designed controllers, each with small, medium, and bigger number of particles and iterations.

The conventional sliding mode control (CSMC) is generally applied to practical systems due to its simple design procedure. In this study, the designed CSMC was similar as the one used in [23]. Furthermore, it was integrated with PSO and GSA in order to allow the adjustment of the controller on these parameters to be conducted automatically. It was also with the purpose for the controller to be adapted to the dynamic changing of the system. The inclusion of CSMC in this study is to illustrate the difference between two types of sliding mode control in the same mismatched system, when result is concerned. As for the number of particles and iterations, these values were chosen in order to prove that the small number of these parameters will result in good tracking performance. The explanation regarding the acquirement of SSE from each combination of CSMC and MSMC with PSO and GSA respectively is displayed in Table 2.

Table 2. SSE obtained from combination of MSMC with PSO and GSA

\begin{tabular}{ccccc}
\hline & \multicolumn{2}{c}{ CSMC } & \multicolumn{2}{c}{ MSMC } \\
& PSO & GSA & PSO & GSA \\
\hline$i 5, t 10$ & 232.1050 & 370.9705 & 32.4511 & 1609 \\
$i 15, t 30$ & 232.1031 & 308.0041 & 32.5014 & 746.6484 \\
$i 25, t 50$ & 232.1030 & 234.5374 & 32.7035 & 881.2971 \\
\hline
\end{tabular}

According to Figure 2, the combination of CSMC and MSMC with PSO and GSA enables the system output to track the reference input with different accuracy. At the beginning, the lowest number of particles and iteration are used which are 5 and 10. Initially, the lowest number of particles and iteration used are 5 and 10. As shown in Table 2, with this combination, MSMC-PSO exhibits the best system output in comparison to the system output of CSMC-PSO, CSMC-GSA, and MSMC-GSA, with SSE amounting to 32.4511, 232.1050, 370.9705, and 1609 respectively. Even though MSMC-PSO produces oscillation at each corner of the output response, the values of SSE are the lowest. Besides, they are capable of tracking the reference input which is accurately provided with small error, in comparison to the capability of others. This indicates that PSO is able to comprehend the optimum combination of the parameter, even with the lowest SSE value. On the contrary, it is apparent that MSMC-GSA displays the most negative performance.

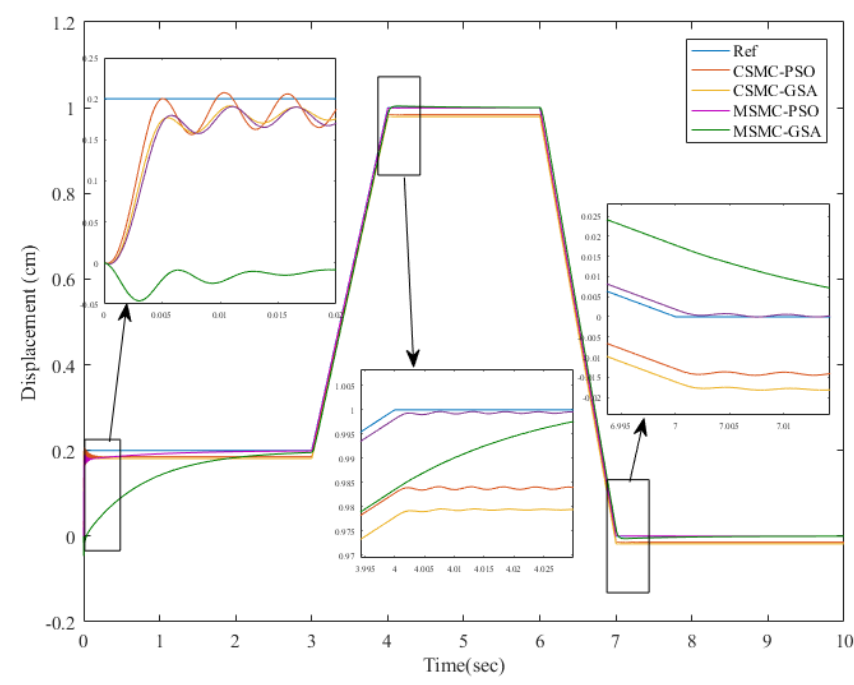

Figure 2. Position output for CSMC-PSO, CSMC-GSA, MSMC-PSO and MSMC-GSA with 5 particles within 10 iterations for external disturbance, $10500 \mathrm{~N}$ 
Figure 3 shows the position output for CSMC-PSO, CSMC-GSA, MSMC-PSO, and MSMC-GSA, with 15 particles within 30 iterations in the presence of external disturbance, which amounts to $10500 \mathrm{~N}$. MSMC-PSO has the tendency to track the provided reference input, with SSE value amounting to 32.5014. However, the oscillation of the output response still occurs at each corner of the position output responses. In comparison to the previous MSMC-PSO output response, no significant change occurs in this situation when the values of $i$ and $t$ are 5 and 10 respectively. Chattering occurs in the output response produced by CSMCPSO and CSMC-GSA. This proves the existence of chattering when conventional sliding mode deals with any system with mismatched nonlinearities. Moreover, the amount of improvement shown by the SSE produced by CSMC-PSO and MSMC-PSO is not significant, despite the increase of the number of particle sand iterations from 5 and 10 to 15 and 30 respectively.

The output response for CSMC and MSMC when the number of particles and iterations is increased to 25 and 50 respectively is illustrated in Figure 4. As seen in Table 2, the decrease of the SSE value for MSMC-GSA occurs, which is from1609, which is significant, to 746. This is followed by its increase to 881 . Despite this increase, these values are still unable to match the performance outputs which is displayed by MSMC-PSO. Besides, the SSE value for MSMC-PSO still amounts to approximately 32, while the SSE values for CSMC-PSO and CSMC-GSA are maintained around 232 and 234 respectively. Provided the emphasis placed on the optimum combination which results to the production of the lowest SSE values for CSMC-PSO, CSMC-GSA, MSMC-PSO, and MSMC-GSA, the optimum combination of the number of particles $(i)$ and iterations $(t)$ is for the best performance output which results in the lowest SSE, as shown in Table 3. Based on Table 3, the combination of MSMC and PSO leads to smaller SSE, in comparison to its combination with GSA. The efficiency of MSMC can be seen through the production of SSE, when CSMC is used as a comparison tool in order to measure the accuracy of MSMC in terms of its management of the mismatched system.

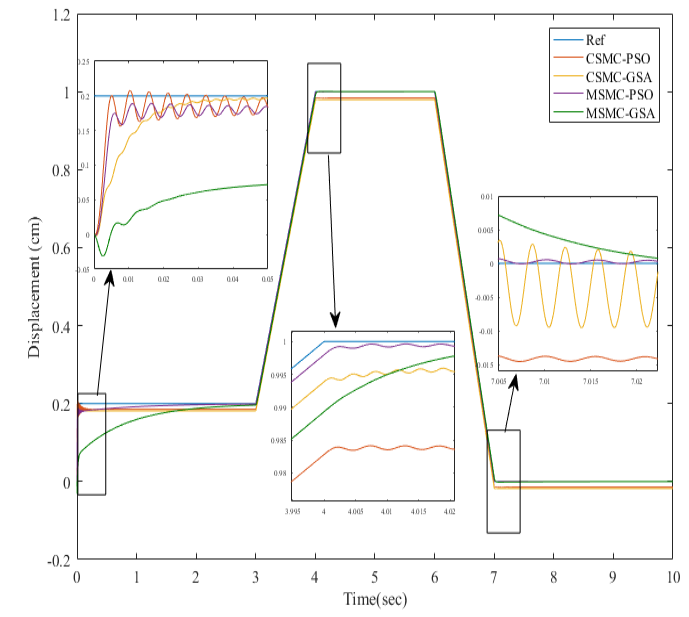

Figure 3. Position output for CSMC-PSO, CSMCGSA, MSMC-PSO and MSMC-GSA with 15 particles within 30 iterations for external disturbance, $10500 \mathrm{~N}$

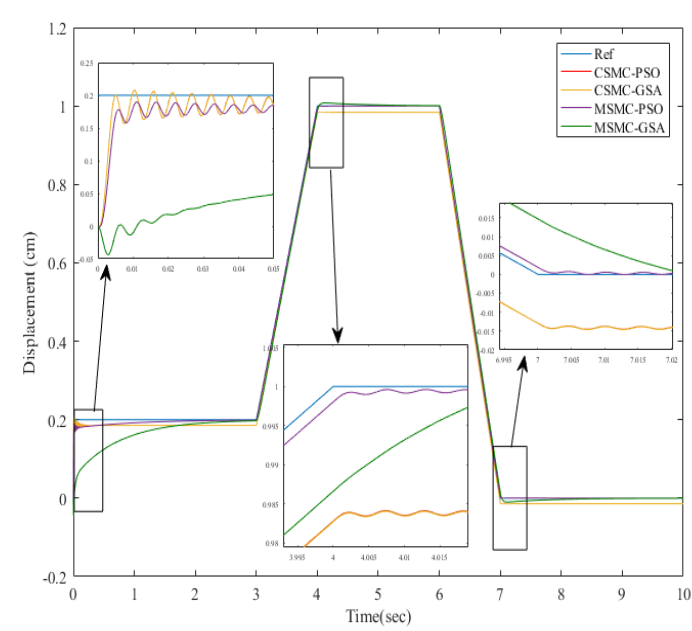

Figure 4. Position output for CSMC-PSO, CSMCGSA, MSMC-PSO and MSMC-GSA with 25 particles within 50 iterations for external disturbance, $10500 \mathrm{~N}$

Table 3. Optimum combination number of particles, $i$ and iterations, $t$ which produced the lowest SSE

\begin{tabular}{ccccc}
\hline & \multicolumn{2}{c}{ CSMC } & \multicolumn{2}{c}{ MSMC } \\
& PSO & GSA & PSO & GSA \\
\hline Number of particles, $i$ & 25 & 25 & 25 & 10 \\
Number of iteration, $t$ & 10 & 50 & 20 & 30 \\
SSE & 232.1030 & 234.5374 & 32.1762 & 743.2693 \\
\hline
\end{tabular}

\section{CONCLUSION}

Based on the results and analysis discussed, the integration of MSMC and PSO produces better tracking performance with small tracking error, in comparison to the combination of the controller similar to GSA's controller and the increasing number of iterations and agents used. With the presence of large external 
disturbance, the integration of MSMC and PSO results in good performance. Besides, this integration results have high accuracy in tracking the reference signal provided to the system. Last but not least, for the next phase of this study, future work which investigates into the techniques required for improvement of the performance output of the proposed control strategy will be put into consideration.

\section{ACKNOWLEDGEMENTS}

The authors would like to acknowledge Universiti Teknologi Malaysia (UTM), Universiti Malaysia Perlis (UNIMAP) and Ministry of Higher Education (MOHE) of Malaysia for their support.

\section{REFERENCES}

[1] C. Kaddissi and J. Kenn, "Identification and Real-Time Control of an Electrohydraulic Servo System Based on Nonlinear Backstepping," IEEE Transactions on Mechatronics, vol/issue: 12(1), pp. 12-22, 2007.

[2] A. A. A. Emhemed, et al., "Modified predictive control for a class of electro-hydraulic actuator," International Journal of Electrical and Computer Engineering, vol. 6, no. 2, pp. 630-638, 2016.

[3] H. M. Kim, et al., "Robust Position Control of Electro-Hydraulic Actuator Systems Using the Adaptive BackStepping Control Scheme," Journal of Systems and Control Engineering, vol. 224, no. 6, pp. 737-746, 2010.

[4] K. K. Ahn, et al., "Adaptive Backstepping Control of an Electrohydraulic Actuator," IEEE/ASME Transactions on Mechatronics, pp. 1-9, 2013.

[5] S. M. Mahdi, "Controlling a Nonlinear Servo Hydraulic System Using PID Controller with Genetic Algorithm Tool," International Journal of Electronics Communication and Computer Engineering, vol. 12, no. 1, pp. 42-52, 2012.

[6] J. Yao, et al., "Extended-State-Observer-Based Output Feedback Nonlinear Robust Control of Hydraulic Systems with Backstepping," IEEE Transactions on Industrial Electronics, vol. 61, no. 11, pp. 6285-6293, 2014.

[7] H. Zhang, et al., "Robust $\mathrm{H} \infty$ sliding mode control with pole placement for a fluid power electrohydraulic actuator (EHA) system," International Journal of Advanced Manufacturing Technology, vol. 73, no. 5-8, pp. 1095-1104, 2014.

[8] S. Wang, et al., "Sliding Mode Controller and Filter Applied to an Electrohydraulic Actuator System," Journal of Dynamic Systems, Measurement, and Control, vol. 133, no. 2, pp. 24504, 2011.

[9] Z. Has, et al., "Robust Position Tracking Control of an Electro-Hydraulic Actuator in the Presence of Friction and Internal Leakage," Arabian Journal for Science and Engineering, vol. 39, no. 4, pp. 2965-2978, 2013.

[10] D. M. Wonohadidjojo, "Position control of electro-hydraulic actuator system using fuzzy logic controller optimized by particle swarm optimization," International Journal of Automation and Computing, vol. 10, 2014.

[11] N. Bouarroudj, et al., "Sliding-Mode Controller Based on Fractional Order Calculus for a Class of Nonlinear Systems," International Journal of Electrical and Computer Engineering, vol. 6, no. 5, pp. 2239-2250, 2016.

[12] H. H. Choi, "LMI-Based Sliding Surface Design for Integral Sliding Mode of Mismatched Uncertain Systems," IEEE Transactions on Automatic Control, vol. 52, no. 4, pp. 736-742, 2007.

[13] I. Ursu, et al., "Backstepping design for controlling electrohydraulic servos," Journal of the Franklin Institute, vol. 343, no. 1, pp. 94-110, 2006.

[14] C. Li, et al., "Projective synchronization of uncertain scale-free network based on modified sliding mode control technique," Physica A, vol. 473, pp. 511-521, 2017.

[15] M. M. Ghanbarian, et al., "Design and implementation of a new modified sliding mode controller for gridconnected inverter to controlling the voltage and frequency," ISA Transactions, vol. 61, pp. 179-187, 2016.

[16] M. Soleymani, et al., "Modified sliding mode control of a seismic active mass damper system considering model uncertainties and input time delay," Journal of Vibration and Control, 2016.

[17] E. Rashedi, et al., "GSA: A Gravitational Search Algorithm," Information Sciences, vol. 179, no. 13, pp. 2232-2248, 2009.

[18] P. K. Hota and N. C. Sahu, "Non-convex economic dispatch with prohibited operating zones through gravitational search algorithm," International Journal of Electrical and Computer Engineering, vol. 5, no. 6, pp. 1234-1244, 2015.

[19] J. Y. Hung, et al., "Variable structure control: a survey," IEEE Transactions on Industrial Electronics, vol. 40, no. 1, pp. 2-22, 1993.

[20] I. M. M. Hassan, et al., "Variable structure control of a magnetic suspension system," Proceedings of the 2001 IEEE International Conference on Control Applications (CCA'01) (Cat. No.01CH37204), pp. 333-338, 2001.

[21] C. S. Chong, "Position tracking optimization for an electro- hydraulic actuator system," Journal of Telecommunication, Electronic and Computer Engineering, vol. 8, no. 7, pp. 1-6, 2016.

[22] S. M. Rozali, et al., "Performance Comparison of Particle Swarm Optimization and Gravitational Search Algorithm to the Designed of Controller for Nonlinear System," Journal of Applied Mathematics, pp. 1-9, 2014.

[23] S. M. Rozali, "Optimized Back-stepping Controller for Position Tracking of Electro-hydraulic Actuators," Phd Thesis, pp. 67-69, 2014. 


\section{BIOGRAPHIES OF AUTHORS}
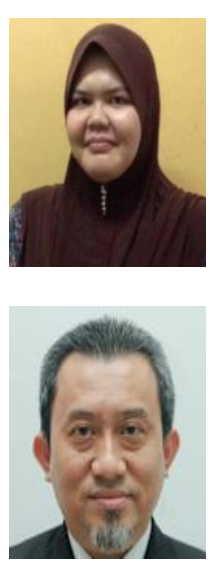

Siti Marhainis Othman received B. Eng. (Mechatronic) from Universiti Teknologi Malaysia in 2006 and M. Eng. (Electrical - Mechatronics and Automatic Control) from Universiti Teknologi Malaysia in 2009. She is currently pursuing $\mathrm{PhD}$ degree in Electrical Engineering at Universiti Teknologi Malaysia. Her main research interest is in the field of nonlinear control system theory.

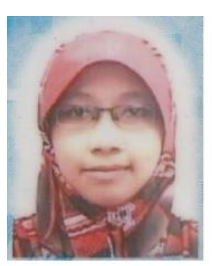

M. F. Rahmat completed B. Eng. (Electrical) from Universiti Teknologi Malaysia in 1989. He obtained the Master degree (Control System Engineering) from The University of Sheffield, UK in 1993 and received a PhD degree in Electronic Instrumentation Engineering from Sheffield Hallam University, UK in 1996. He is currently a Professor in the Department of Control and Mechatronics Engineering, Faculty of Electrical Engineering, Universiti Teknologi Malaysia, Skudai, Johor. He has also now been appointed as a Dean of UTM Space, Skudai, Johor. His field of specialization includes system identification, signal processing, process tomography, process control and instrumentation, sensors, and actuators.

Sahazati Md. Rozali received B. Eng (Hons.) (Electronic Engineering) from the Universiti Sains Malaysia, Malaysian in 2004, Master of Engineering(Electrical-Mechatronics and Automatic Control) from Universiti Teknologi Malaysia in 2007 and $\mathrm{PhD}$ in Electrical in 2014. She is currently a Senior Lecturer at Department of Electrical Engineering Technology, Faculty of Engineering Technology, Universiti Teknikal Malaysia Melaka. Her field of specialization are control system and system identification.

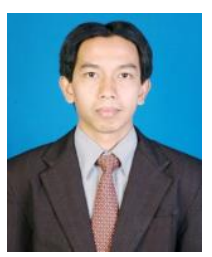

Zulfatman completed B. Eng. (Electrical) from University of Muhammadiyah Malang in 2003. He received the Master degree and a $\mathrm{PhD}$ degree in Control System Engineering from Universiti Teknologi in 2009 and 2015. He is currently a Senior Lecturer in the Department of Electrical Engineering, Faculty of Engineering, University of Muhammadiyah Malang, Indonesia. His area of research includes control systems for renewable energy generation and early warning systems.

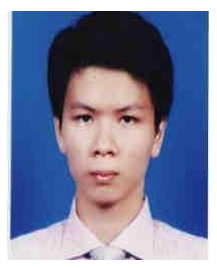

Amar Faiz Zainal Abidin received his Bachelor of Engineering in Electrical \& Electronics from University of Nottingham in 2008. While working as Tutor in Universiti Teknologi Malaysia (UTM), he completed his master degrees: Master of Engineering in Electrical (Mechatronics \& Automatic Control) from UTM and Master of Science in Computer Vision from University of Burgundy. Currently, he serves Universiti Teknikal Malaysia Melaka as a Lecturer and his main research interest is in Computational Intelligence 